\title{
Puzzlemote: videojuego controlado con el mando de la Wii para niños de 6 a 10 años
}

Marcelo Daniel Torres Vinueza, Walter Fuertes, César Xavier Villacís Silva, Margarita Elizabeth Zambrano Rivera, Carlos Teiron Prócel Silva

\begin{abstract}
Resumen
Introducción: Los videojuegos educativos ayudan a crear situaciones de aprendizaje significativo por descubrimiento, que puede aportar habilidades de resolución de problemas y toma de decisiones. Esta investigación presenta el diseño, implementación y evaluación de un Software Educativo basado en videojuegos denominado Puzzlemote, enfocado a niños de 6 a 10 años. Método: Se ha empleado la Metodología de Diseño Hipermedia Orientada a Objetos (OOHDM) para crear un ambiente lúdico e interactivo y a la Inteligencia Artificial (IA) que promueve el razonamiento lógico y la resolución de problemas complejos. El algoritmo utilizado implementa las técnicas de razonamiento hacia adelante y hacia atrás tanto para desordenar el rompecabezas como para ordenarlo, lo cual difiere de otros del mismo tipo, donde lo común es utilizar el Algoritmo $A^{*}$. Para su ejecución se ha recurrido a la manipulación del control remoto de la consola Wii (Wiimote) para usarlo durante el tiempo de juego, lo cual permite al usuario tener una experiencia más completa con el software que está conectado a dicho dispositivo. Resultados: Los resultados muestran que este programa mejora el raciocinio lógico y comprensión de solución de problemas, usando los rompecabezas conocidos como n-puzzle, a niños que se encuentran en su etapa primaria de enseñanza. Conclusiones: Se pudo comprobar que la combinación de las técnicas de razonamiento utilizando grafos facilita que la computadora desarme y arme el rompecabezas con jugadas válidas, memorizando los movimientos iniciales y reconstruyendo esos movimientos invirtiendo la lista enlazada. Como trabajo futuro se plantea desarrollar este videojuego para dispositivos móviles y plataformas 3D con otras consolas de videojuegos tales como Play Station, Nintendo y X-Box. Además, se planea trascender con este tipo de tipo de aplicaciones, también para personas de la tercera edad ya que ayudan al entrenamiento de la memoria y podrian evitar el desarrollo del Síndrome de Alzheimer.
\end{abstract}

\section{Palabras clave}

Videojuegos. Juegos educativos. Metodología de Diseño Hipermedia Orientada a Objetos. Inteligencia artificial. Wii.

\section{Introducción}

Los videojuegos didácticos posibilitan la interactividad con los estudiantes, facilitan las representaciones animadas y permiten simular procesos complejos. Abren nuevas posibilidades de innovación e implementación de nuevos modelos que facilitan el proceso de enseñanza. Por otro lado, el aprendizaje del uso dispositivos de tecnología como computadoras, celulares, video juegos, etc., se hace cada vez más intuitivo, tanto así que los niños de ahora desarrollan con facilidad el conocimiento y la habilidad necesaria para manejarlos (Kurzweil, 2010). Sin embargo, la industria de este software en el Ecuador no se ha explotado suficientemente, especialmente en el ámbito educacional para niños de temprana edad, ya que el costo de desarrollar estas herramientas puede ser elevado.

Ante este escenario, en el ámbito educativo, algunas investigaciones (Murphy, Penuel, Means, Korbak \& Whaley, 2001), (González \& Zapata, 2010), (Álvarez, 2013), (Quishpe \& Conde, 2010), han demostrado que los juegos y el software educativo desarrollan destrezas integrales para lograr competencias en el uso y manejo de la información por parte de los estudiantes. Otros trabajos han demostrado que el software educativo proporciona al profesor la posibilidad de 
crear nuevos contenidos y adaptarlos a las características de los estudiantes (Barajas \& Álvarez, 2012; Rodríguez, Pompa, Rodríguez, \& Hidalgo, 2013). Resulta oportuno citar a López (2011) que ha verificado la relación entre el consumo de videojuegos y el rendimiento académico. En trabajos más recientes Marzal y Sáez (2013) y Illanas (2013) exponen investigaciones relacionados con los nuevos desafíos de los videojuegos, las multi-disciplinas, las promesas genealógicas en la retórica publicitaria de PlayStation, mecánicas de juego en los dispositivos móviles, realidad virtual e Inteligencia Artificial (IA). Finalmente en Lee (2008), se desarrollan videojuegos con el uso del control remoto de la consola Wii.

En relación con la temática de nuestro trabajo de investigación, Charles Babbage, arquitecto de computadoras del siglo XIX, ya pensaba en programar juegos como el ajedrez y el tres en raya. Más tarde Arthur Samuel, a principios de los años 60 construyó el primer juego de damas. Otros juegos que generan interés en la gente son los puzzles (rompecabezas) como el 8-puzzle, 15-puzzle y 24-puzzle, entre otros (Levitin \& Levitin, 2011). El problema del 8-puzzle fue discutido por Doran y Michie (1966). Para dar continuidad, Korf (1999) ha dado una visión general de los problemas de la n-puzzle. El 15-puzzle puede ser resuelto con el algoritmo de IDA* (iterative deependig $A^{\star}$ ) propuesto por Korf (1985) que fue el primero en obtener una solución óptima de este problema. Más adelante, Korf y Schultze (2005) proporcionaron una mejora a los algoritmos de búsqueda del problema del 15-puzzle (Millington, 2009), (Buckland, 2004).

Sobre la base de las consideraciones anteriores, se puede apreciar el interés por la comunidad científica en el desarrollo, aplicación e investigación de video juegos educativos, sobre todo con el uso de técnicas de IA que permite que los videojuegos provoquen un inexplicable encanto y fascinación. Por tanto se desea comprobar que si las computadoras puedan jugar y simular la presencia de contrincantes, entonces los videos juegos pueden incrementar el razonamiento lógico y la comprensión.
El presente proyecto tiene como objetivo diseñar e implementar Puzzlemote, un videojuego didáctico enfocado a niños de 6 a 10 años. Para llevarlo a cabo se inició con la investigación descriptiva de videojuegos didácticos y motores de juegos. Seguidamente, se cumplió las fases de la Metodología de Diseño Hipermedia Orientada a Objetos (OOHDM) y su aplicabilidad para el desarrollo de videojuegos. Así mismo se investigó la utilización de las técnicas de razonamiento de Inteligencia Artificial (IA) llamadas encadenamiento hacia atrás y hacia adelante. Luego mediante el lenguaje unificado de modelamiento (UML) se modeló la aplicación $2 \mathrm{D}$, la técnica de IA señalada, y la librería para la manipulación del Control Remoto de la Consola Wii (Wiimote) para usarlo durante el juego. Finalmente se implementó la interfaz en dos dimensiones, que fue controlada por este dispositivo y por las reglas de razonamiento provisto por la IA. Los resultados muestran que este programa mejora el raciocinio lógico y comprensión de solución de problemas, al tener una experiencia más completa con el software que está conectado a dicho mecanismo, empleando los rompecabezas conocidos como n-puzzle, a niños que se encuentran en su etapa primaria de enseñanza.

Como contribución se ha logrado interactuar con el Wiimote, el cual permite conectarse a un equipo por medio de tecnología Bluetooth y realizar una acción sobre un software educativo con el movimiento de las manos. Adicionalmente, el algoritmo programado, difiere de otros del mismo tipo, donde lo común es utilizar el Algoritmo $A^{*}$ de IA, mientras que aquí se utilizó las técnicas de razonamiento hacia adelante y hacia atrás tanto para desordenar el rompecabezas como para ordenarlo.

El resto del artículo ha sido organizado como sigue: La sección 2 describe el fundamento teórico que sustenta esta investigación. En la sección 3 se explica el diseño conceptual, diseño navegacional, diseño abstracto de interface y la implementación del videojuego. En la sección 4 se ilustran los resultados obtenidos. En la sección 5 se analizan los trabajos relacionados. Finalmente en la 
sección 6 se exponen las conclusiones y el trabajo futuro.

\section{Marco referencial}

En esta sección se describe una serie de elementos conceptuales mediante los cuales se desarrolló este proyecto de investigación.

\section{Video juegos y aprendizaje}

En nuestro proyecto se ha tomado como base el estudio de Álvarez (2013) quien establece cinco especificaciones claves de los videojuegos para su desarrollo y ejecución; deben: ser enfocados en una sola competencia; tener una interfaz de usuario gráfica simple y evaluada pedagógicamente; exhibir casos con razonamiento formal; mostrar casos generados aleatoriamente para prevenir que el estudiante memorice las respuestas a los problemas; $y$ tener contenido retador y generador de competitividad entre los estudiantes que usen el videojuego; es decir, caos con diferentes niveles de dificultad. Por otro lado, se debe reconocer que los videojuegos constituyen un fenómeno popular asociado con el desarrollo tecnológico, que ha ampliado su campo de acción y sobrepasado la frontera de entretenimiento, alcanzando el ámbito educativo.

Uno de los tipos de videojuegos es el rompecabezas, que es un problema o enigma que se debe solventar por medio de algún tipo de genialidad. En un rompecabezas básico, se debe colocar con lógica las piezas o fichas en orden, de tal manera que se encuentre una solución deseada para el problema. Entre los más comunes de estos juegos está la n-puzzle. El n-puzzle consiste en un tablero $\mathrm{NxN}$ casillas, $\mathrm{N}-1$ de las casillas contienen una ficha que se puede deslizar por el tablero de manera horizontal (izquierda y derecha) y vertical (arriba y abajo). Las fichas están marcadas con número del 1 al $\mathrm{N}$ y se cuenta con una casilla en blanco que permite los movimientos de las fichas. El problema es llegar a un estado final o meta en el que la disposición de las fichas está ordenada. En nuestro proyecto se aplica el 15-puzzle desarmado, en el cual se debe mover las fichas alrede- dor del espacio vacío, de tal manera que vayan quedando en orden y con el espacio vacío al final.

\section{Inteligencia Artificial para Videojuegos}

La Inteligencia Artificial (IA) es un campo de las ciencias computacionales que se puede aplicar en el proceso de enseñanza aprendizaje, que fomenta el razonamiento lógico, para resolver problemas complejos y encontrar soluciones de una manera más rápida y segura. La IA otorga a la computadora la capacidad de aparentar un raciocinio humano (León, 2008), y logra el dominio del aprendizaje por el reforzamiento y ejercitación, favorece procesos de construcción de conocimiento, reconoce una extensa gama de errores de razonamiento, provee conjuntos de problemas distintos y gradúa la dificultad relativa (Bello, 2002), (Rich \& Kevin, 1994).

En este proyecto se ha seleccionado la combinación de técnicas de razonamiento hacia adelante y hacia atrás, que consiste en construir un árbol de secuencias de movimientos que ofrezcan soluciones empezando con las configuraciones objetivo en la raíz del árbol. Luego se generará el siguiente nivel del árbol encontrando todas las reglas cuyos lados derechos estén ligados con el nodo raíz. Éstas serán todas las reglas que, si son las únicas que se aplican, generarán el estado que se desea. Se utilizará el lado izquierdo de las reglas para generar los nodos en este segundo nivel del árbol (Rusell \& Meter, 1994).

\section{Metodología de Diseño Hipermedia Orientada a Objetos}

La OOHDM es una metodología de diseño de hipermedia, que utiliza el enfoque orientado a objetos, con técnicas de representación gráfica de relaciones entre objetos y de contextos navegacionales que proveen representación estructural y semántica (Silva \& Mercerat, 2001). Esta metodología se basa en cuatro etapas: diseño conceptual, diseño navegacional, diseño abstracto de interface e implementación. Ha sido escogida en esta investigación porque reúne las características necesarias para distinguir aspectos concep- 
tuales (modelo del dominio) de su presentación (construcción de la interfaz de usuario). La metodología OOHDM es apropiada para desarrollo de sistemas complejos (Shneiderman \& Plaisant, 2006). Las ventajas que ofrece son una clara identificación de los diferentes niveles de diseño en forma independiente de la implementación, y la forma gráfica que se usa para representar los diseños. Además se integra a la arquitectura Modelo Vista Controlador y permite una planificación más adecuada para el desarrollo de un sistema informático (Durkin, 1994).

\section{Wiimote}

Es el control remoto de la consola Wii de Nintendo, el cual también es compatible con la consola Wii-U. Sus características son la capacidad de detección de movimiento en el espacio y la habilidad de apuntar hacia objetos en la pantalla. Su diseño es similar a un control remoto de televisión, creado para ser utilizado en una sola mano y de la manera más intuitiva posible y está construido alrededor del chip de Bluetooth Broadcom (Selver 2004), (Lee, Hudson \& Tse, 2008).

\section{Visual Studio y Librería Gráfica GDI+}

En este proyecto, Microsoft Visual Studio provee los IDE para el desarrollo de aplicativos de consola, programas para usuario con interfaces gráficas, aplicaciones con formularios, servicios Web y la combinación con otras plataformas desarrolladas por Microsoft. Además se ha utilizado la librería gráfica de Windows GDI+ (Graphics Device Interfaces+) que permite al Sistema Operativo la construcción de gráficos independiente de la marca de la tarjeta de video.

\section{Diseño e implementación}

En esta sección se muestra cómo se diseñó e implementó el videojuego didáctico Puzzlemote. El proyecto constó de tres tipos de rompecabezas, seis animales para armar, conexión y reconocimiento con control remoto del Wii, archivos planos para manejo de información, módulo de seguridad de producto y aplicación de la IA para armado y desarmado.

\section{Modelo IA: caso Puzzlemote}

Para el modelo de IA de la aplicación se ha empleado la combinación de las técnicas de razonamiento hacia delante y hacia atrás, con lo cual se utiliza grafos dirigidos, que son representados por listas enlazadas. Estas se encargan de almacenar los diferentes movimientos hechos por la misma aplicación y el usuario. Desde a un estado inicial al desordenar el rompecabezas, se puede volver al estado objetivo, donde la aplicación puede reproducir todas las jugadas al manejar los nodos de la lista enlazada almacenando los movimientos del estado inicial y luego invertir la lista para llegar u obtener el estado objetivo. En la Figura 1 se muestra tanto los valores iniciales y finales de la lista de fichas, como la lista de valores que guardan los movimientos.

Figura 1 - Índice de Posiciones en un Puzzle
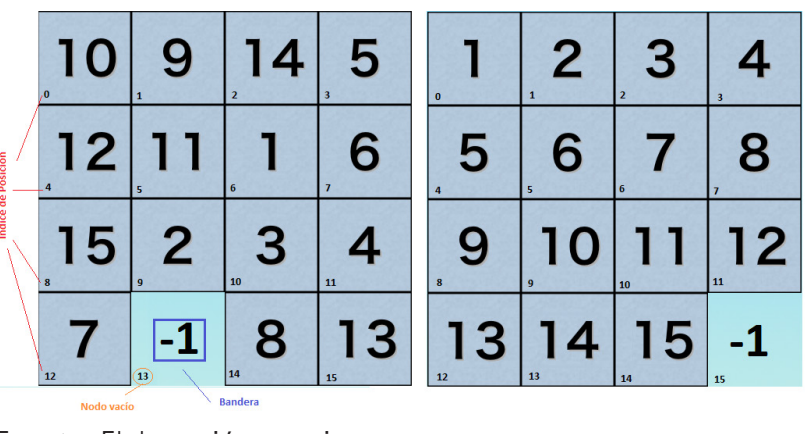

Fuente: Elaboración propia.

Este modelo detalla cómo se encuentra estructurado un rompecabezas para 15 fichas. Se compone de 15 grafos dirigidos, los cuales representan un grupo de movimientos válidos que se pueden realizar dentro de la aplicación. Así por ejemplo, el nodo vacío, que en la Figura 1 es el 13, solo puede relacionarse con los nodos 9, 12 y 14 (índices de posición), es decir sus nodos vecinos, de tal manera que se obtiene un grafo que se puede representar con la siguiente ecuación:

$G_{10}=((13,9),(13,12),(13,14))$

Ecuación (1)

Como se puede apreciar, en el modelo se utiliza una bandera con el valor de -1, la cual indica la ubicación del espacio de la ficha vacía. Cuando se mueve una ficha en el rompecabezas, sucede 
que los contenidos de ambas fichas (la vacía y la ocupada) intercambian el dato de su número, convirtiendo la ficha vacía en visible y la ocupada en invisible; de tal manera que al final del juego, la ficha vacía se debe encontrar en la posición 15 (la última ficha que está en el tablero de juego). En el caso del juego, la posición 15 corresponde a un botón o controlador de imagen.

La información que se muestra en las Figuras 2 y 3 , se refiere a los viables movimientos válidos de las fichas dentro de los posibles rompecabezas de la aplicación $(8,15 \mathrm{e})$, representados por grafos dirigidos:

Figura 2 - Grafos Rompecabezas 8

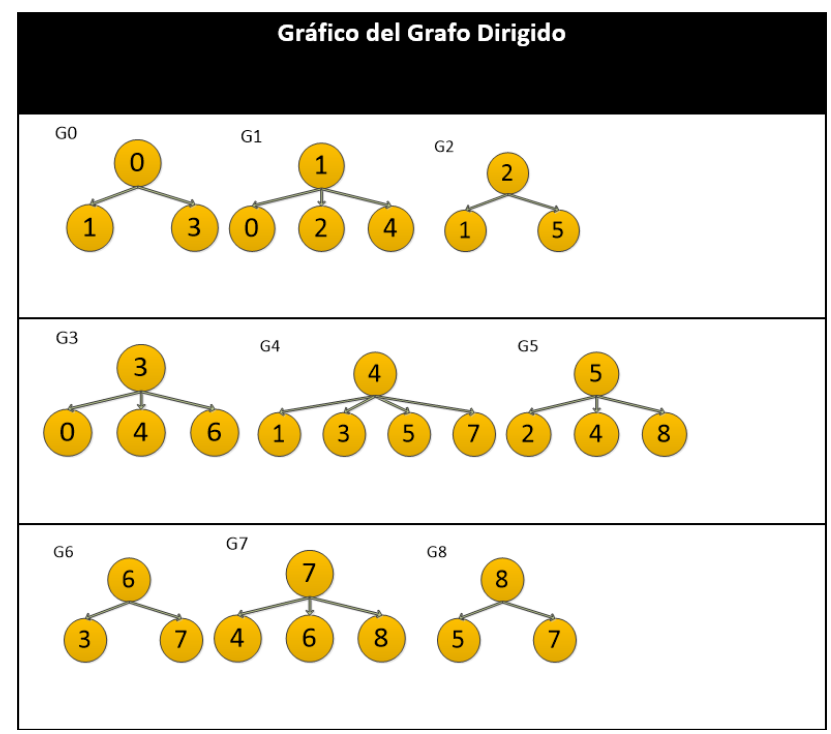

Fuente: Elaboración propia.

Figura 3 - Grafos Rompecabezas 15

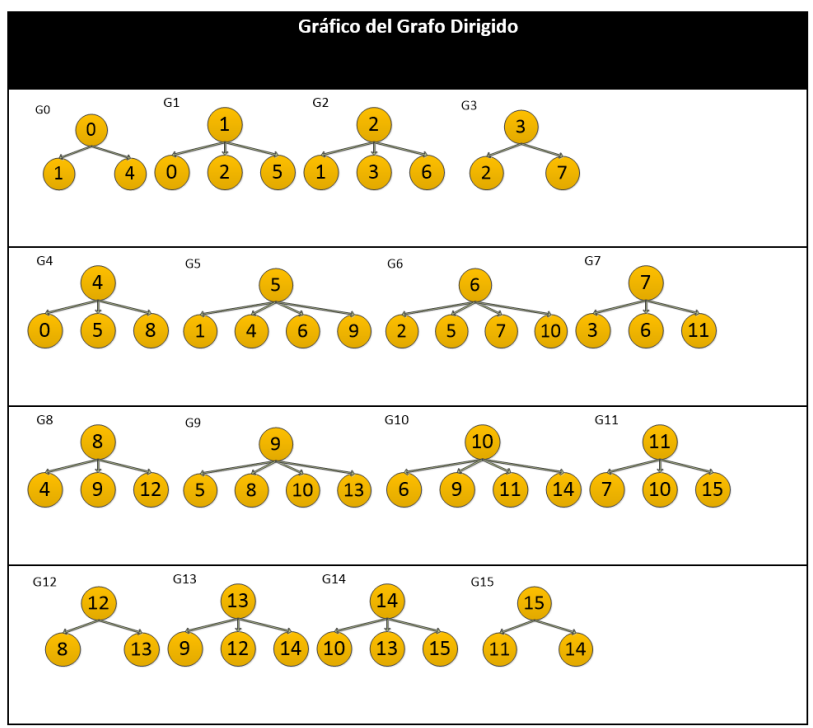

Fuente: Elaboración propia.

\section{Especificación de requerimientos}

El sistema tuvo que cumplir las siguientes tareas y roles:
a) iniciar programa;
b) activar el producto;
c) configurar el juego;
d) mover una ficha en una partida del jue- go;
e) desplegar el puntaje de los juegos;
f) seleccionar nueva imagen
g) armar solo el rompecabezas;
h) cambiar el nombre del jugador; $y$
i) evaluar y salir del juego.

Las Figuras 4 y 5, muestran los diagramas de casos de uso de la aplicación Puzzlemote y uno de

Figura 4 - Casos de uso del sistema Puzzlemote

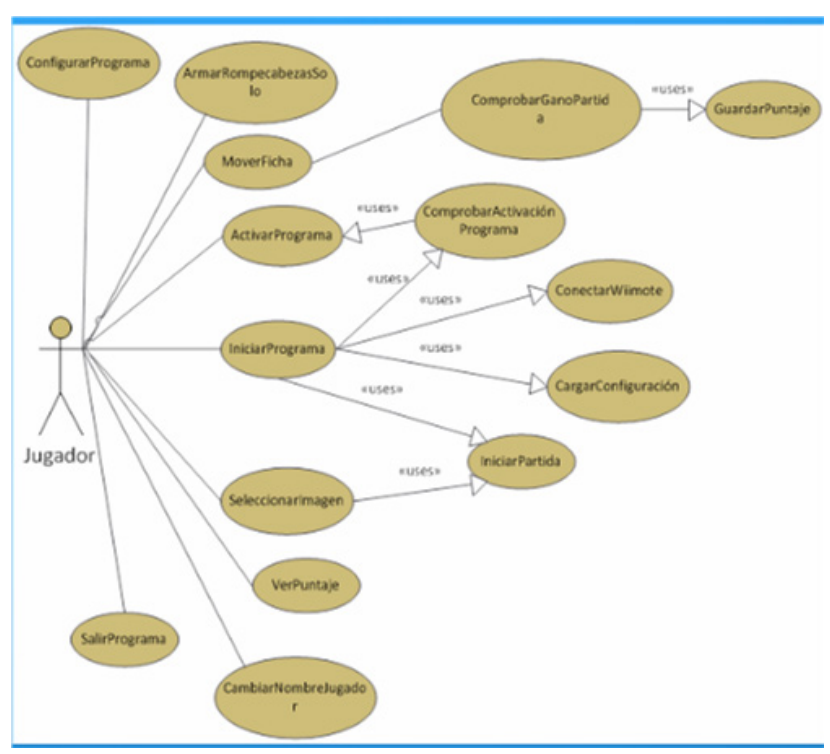

Fuente: Elaboración propia.

Figura 5 - Diagrama de Secuencias "Mover una ficha con control Wii"

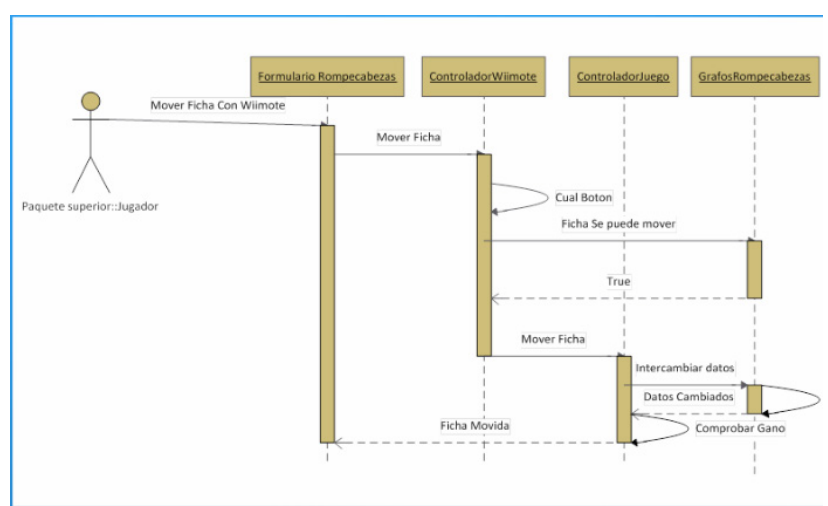

Fuente: Elaboración propia. 
secuencias de cómo mover una ficha con control Wii.

\section{Diseño Conceptual}

En esta fase, se diseñaron e implementaron archivos planos para la manipulación y almacenamiento de información, como son puntajes, datos referenciales, configuraciones y usuarios del sistema. Así mismo, se determinó que la arquitectura a utilizar sería el Modelo Vista-Controlador, el cual se complementa con OOHDM, separando el diseño de interfaz con el motor-control del juego. El sistema posee cinco (5) capas de control las cuales son: controlador del juego, controlador de registro del sistema, controlador de seguridad, controlador de la librería Wiimote y vista de sistema.

\section{Modelo Navegacional}

En esta fase, el desarrollo de las interfaces estuvo marcado por el uso de formularios (los controles Form), los que permiten una adecuada forma para desarrollar vistas, además de ser estéticamente acertadas. Los objetos Navegacionales son: control de programa, formularios de rompecabezas 8-puzzle, 15-puzzle, 24-puzzle y formulario de activación de producto. Los contextos navegacionales son: seleccionar imagen, configurar juego, acerca del juego, ayuda del juego, puntajes.

\section{Implementación y pruebas}

\section{Construcción de Componentes Puzzlemote}

En los Cuadros 1, 2 y 3 se describen los principales componentes, sus clases y su descripción que se encargan de la especificación de requerimientos expuestos en el proyecto.

\section{Construcción de la Interfaz Puzzlemote}

La interfaz se divide en una clase (Program), que se encarga de mostrar la ventana adecuada de acuerdo a la configuración guardada del sistema; y tres formularios que corresponden a cada nivel que posee el juego (el formulario frmRompecabezas9 de 8-puzzle, el frmRompecabezas16 de 15-puzzle y el frmRompecabezas 25 de 24-puzzle). Los 3 formularios a su vez contienen "sub-ventanas", hechas con el Control GroupBox, que muestran la "Configuración del Juego", la ventana "Acerca del Juego, y dentro de esta, la opción de levantar la animación de ayuda creada en el programa Prezi. Las figuras 6 a 8 muestran el funcionamiento del videojuego didáctico y su ejecución:

Cuadro 1 - Componente Librería Wiimote

$\begin{array}{lll}\text { ComponenteLibreriaWimote } & \text { Clases } & \text { Descriptión } \\ \begin{array}{lll}\text { Es el componente que se } \\ \text { encargará de la contención de } \\ \text { datos de Wiimote disponible y } \\ \begin{array}{l}\text { conectado con la PC. Posee las } \\ \text { clases: }\end{array}\end{array} & \text { WiiMote } & \text { Contiene los estados que presenta un Wiimote conectado al aplicativo } \\ & & \begin{array}{l}\text { Los enumeradores son estructuras de datos, permiten almacenar } \\ \text { números estáticos que repiten constatemente durante el desarrollo del } \\ \text { software, facilitando su uso dentro del código }\end{array}\end{array}$

Fuente: Elaboración propia.

Cuadro 2 - Componente Juego

\begin{tabular}{|c|c|c|}
\hline ComponenteSeguridades & Librerias & Descriptión \\
\hline $\begin{array}{l}\text { Se encarga de manejar la } \\
\text { activiación o no del producto } \\
\text { Puzzlemote instalado }\end{array}$ & TrialMaker & $\begin{array}{l}\text { Hace uso de la una libreria de control libre baseada en C\# Ilamada } \\
\text { "TrialMaker", el cual posee los componentes necessarios para el manejo } \\
\text { de ventanas y arquivos en el registro de seguridad de activación }\end{array}$ \\
\hline
\end{tabular}

Fuente: Elaboración propia. 


\section{Cuadro 3 - Componente Seguridades}

\begin{tabular}{|c|c|c|}
\hline ComponenteJuego & Clases & Descriptión \\
\hline \multirow{12}{*}{$\begin{array}{l}\text { Es el componente } \\
\text { "motor" o "corazón" } \\
\text { de la aplicación, posee } \\
\text { el cerebro del juego, el } \\
\text { comportamiento de los } \\
\text { formularios, el control de } \\
\text { sonido, de cronómetros } \\
\text { y movimientos, el } \\
\text { manejo de los archivos } \\
\text { planos y finalmente } \\
\text { conecta y controla el } \\
\text { comportamiento de un } \\
\text { Wiimote conectado a la PC } \\
\text { por medio del componente }\end{array}$} & VistaJuego & Controla la vista del juego \\
\hline & Tiempo & Controla los movimentos y el tiempo del sistema \\
\hline & Sonido & Controla el sonido del sistema \\
\hline & GrafosRompecabeza & $\begin{array}{l}\text { Controla los movimientos que maneja el sistema. Esta clase es la que } \\
\text { implementa el razonamiento "hacia adelante" y "hacia atrás" }\end{array}$ \\
\hline & ClaseGlobalJuego & Controla los atributos globales que tiene el sistema \\
\hline & Animal & Cotiene la información de cada animal del juego \\
\hline & ArchivoPlano & Controla el guardado de datos, como configuraciones y puntajes \\
\hline & CerebroJuego & $\begin{array}{l}\text { Indica como se debe armar, como hacer los movimientos y cuando se } \\
\text { gana el juego }\end{array}$ \\
\hline & ControladorJuego & $\begin{array}{l}\text { Controla la vista, el armado, los movimientos y cuando se gana en el } \\
\text { sistema. Esta es la clase que aglutina toda la lógica del juego }\end{array}$ \\
\hline & PuntajeUsuario & Controla el puntaje de los jugadores en el sistema \\
\hline & ControladorWiimote & Controal el estado y el manejo del control Wiimote en el sistema \\
\hline & Parametros & $\begin{array}{l}\text { Es una clase estática donde se guardan datos estáticos que se usarán } \\
\text { durante todo el programa }\end{array}$ \\
\hline
\end{tabular}

Fuente: Elaboración propia.

Figura 6 - Interfaz del Rompecabezas de 15 piezas

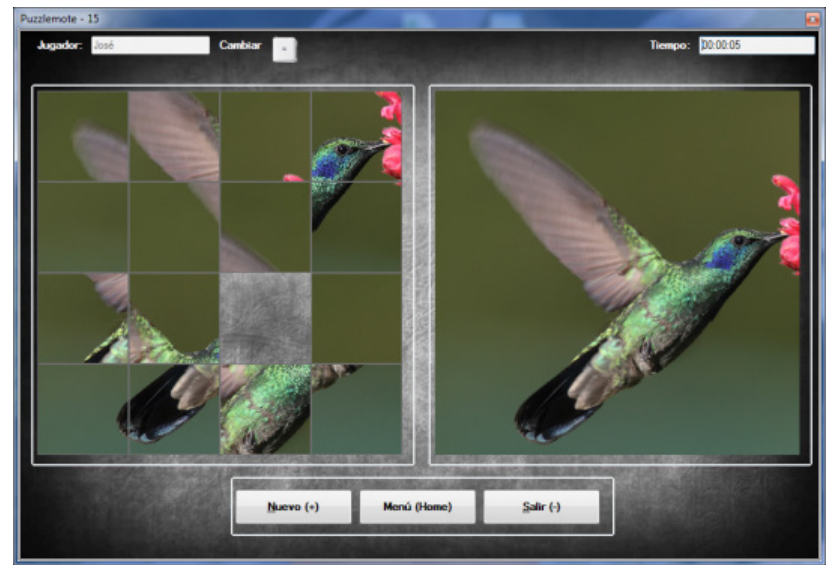

Fuente: Elaboración propia.

Figura 7 - Interfaz del Nuevo juego

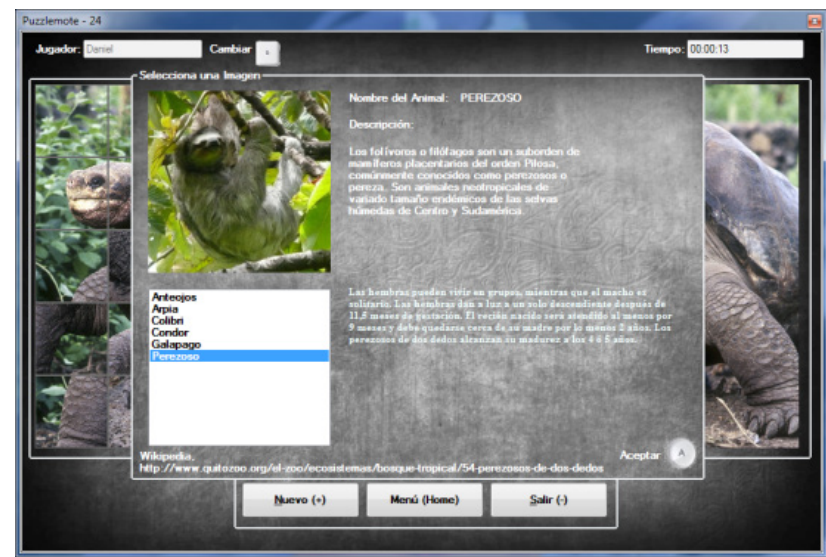

Fuente: Elaboración propia.
Figura 8 - Interfaz del Menú de Configuración

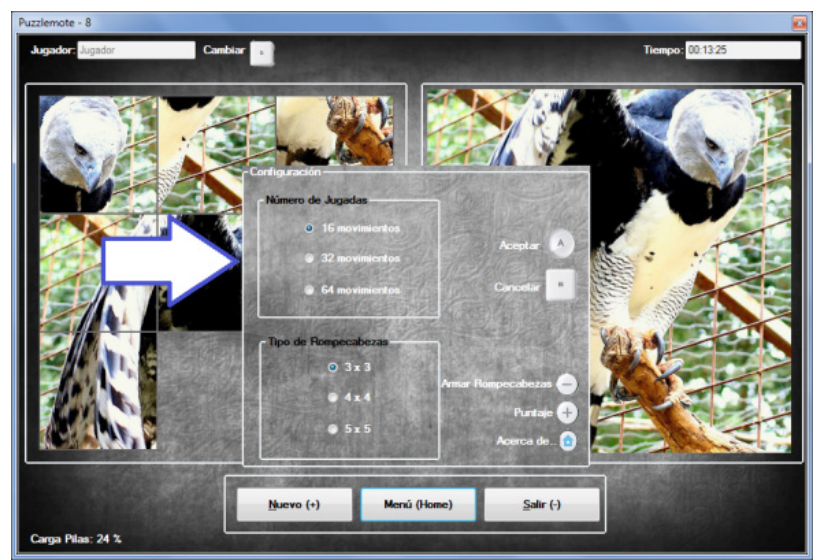

Fuente: Elaboración propia.

Figura 9 - La interfaz del puntaje obtenido

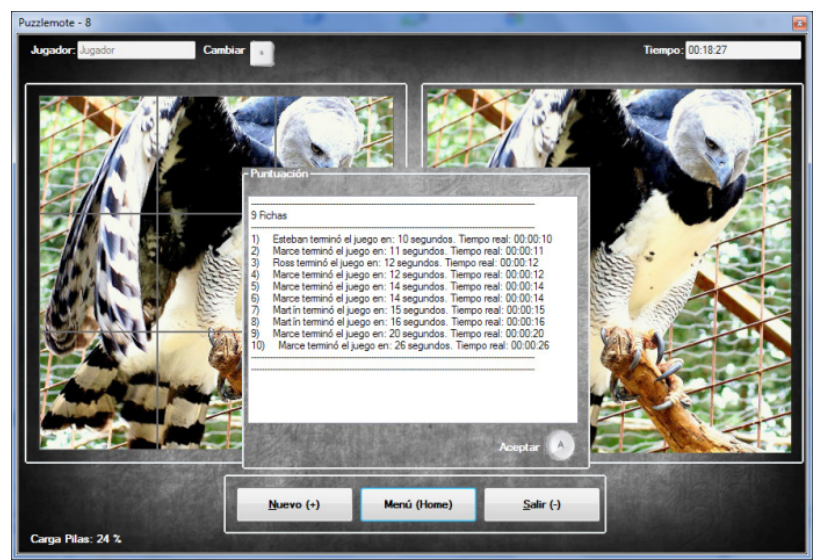

Fuente: Elaboración propia.

Curitiba, v. 2, n. 2, p. 94-105, jul./dez. 2013 | www.atoz.ufpr.br 
Los formularios del juego poseen la misma estructura de diseño (Tabla 2), la cual se divide en: control de nombre de jugador (el cual posee un control para cambiar el nombre); contenedor del tiempo transcurrido en una partida; contenedor del rompecabezas; imagen de ayuda para armar el rompecabezas; y, controles del juego (nuevo juego, menú de configuración y salir del juego).

Puzzlemote tiene derechos reservados en la empresa Virtual Learning Solutions, y se puede visualizar el Manual de usuario en línea, en el URL http://prezi.com/zg4dpdt9jb3o/Puzzlemote-manual-de-usuario/.

\section{Evaluación de Resultados}

Para probar la aplicación se seleccionó como proyecto piloto una escuela privada del cantón Quito, para lo cual se tomó una muestra de 18 alumnos de una población total de 270 , que corresponden a los grupos de niños entre tercero y quinto grado de educación básica, donde cada grupo tiene un total de 90 estudiantes distribuidos en tres paralelos (A, B, C). De este análisis se pudo determinar los siguientes resultados:

a) para el 8-Puzzle solamente un grupo de 10 estudiantes, según la muestra, lograron resolver el rompecabezas en el segundo intento, luego de proveerles las pautas básicas para solucionarlo, dentro de un tiempo de cinco minutos;

b) para el 15-Puzzle solamente un grupo de 4 estudiantes, según la muestra, lograron resolver el rompecabezas en el segundo intento, luego de darles, las pautas básicas para solucionarlo, dentro de un tiempo de cinco minutos; $y$

c) para el 24-Puzzle, no hubo ningún interés por parte de los estudiantes para resolverlo, ya que resultaba complejo completarlo.

Estos resultados se muestran en las Tablas 1 y 2 de acuerdo al género y grado seleccionados.
Tabla 1 - Resultados del 8-Puzzle

\begin{tabular}{lcccc}
\hline \multirow{2}{*}{ Niños } & \multicolumn{2}{c}{ Masculino } & \multicolumn{2}{c}{ Feminino } \\
\cline { 2 - 5 } & Total & Porcentaje & Total & Porcentaje \\
\cline { 2 - 5 } Tercer Grado & 0 & 0 & 2 & 33,33 \\
Cuarto Grado & 2 & 50 & 2 & 33,33 \\
Quinto Grado & 2 & 50 & 2 & 33,33 \\
Total & 4 & 100 & 6 & 100 \\
\hline
\end{tabular}

Fuente: Elaboración propia.

Tabla 2 - Resultados del 15-Puzzle

\begin{tabular}{lcccc}
\hline Niños & \multicolumn{2}{c}{ Masculino } & \multicolumn{2}{c}{ Feminino } \\
\cline { 2 - 5 } & Total & Porcentaje & Total & Porcentaje \\
\cline { 2 - 5 } Tercer Grado & 0 & 0 & 0 & 33,33 \\
Cuarto Grado & 0 & 50 & 1 & 33,33 \\
Quinto Grado & 1 & 50 & 2 & 33,33 \\
Total & 1 & 100 & 3 & 100 \\
\hline
\end{tabular}

Fuente: Elaboración propia.

\section{Trabajos relacionados}

Durante la investigación se han encontrado una importante cantidad de trabajos relacionados con los videojuegos didácticos. A continuación se resume el estado del arte:

En el trabajo presentado por González y Zapata (2010) se desarrolló un software educativo adaptado a las necesidades de los niños y docentes, con características de interactividad, presencia de elementos multimedia, para desarrollar habilidades. En este mismo contexto, Quishpe y Conde (2010) investigaron cómo incide el uso de software multimedia en el aprendizaje de los niños con necesidades educativas especiales. Sus resultados muestran asistencias pedagógicas ya que el aprendizaje por medio de un computador sirve de motivación hacia los niños con necesidades educativas especiales. En Rodríguez (2013) se presenta la implementación de una aplicación dirigida a la enseñanza primaria, la cual apoya el proceso de enseñanza-aprendizaje, a la vez que le proporciona al profesor la posibilidad de crear nuevos contenidos y adaptarlos a las características de los estudiantes. En López (2011) se presenta un estudio que demuestra una correlación negativa de muy baja intensidad entre el grado de atracción por los videojuegos y el rendimiento académico, no produciéndose en todas las áreas. 
En Marzal y Sáez (2013) se presenta un libro titulado Videojuegos y Cultura Visual, que recoge diferentes aproximaciones al estudio de los videojuegos desde las formas de producción de sentido de los videojuegos, los hábitos de consumo y su construcción narrativa en el contexto de la cultura digital, evaluando la realidad empresarial que se está construyendo alrededor de esta industria.

En relación a videojuegos que permiten realizar investigación con fines educativos en Illanas (2013) y Illanas, Calvo-Ferreret, Gallego y Llorens (2013), se persigue el objetivo de que el jugador se convierta en un intérprete reconocido y, para ello, debe ser capaz de completar las traducciones en castellano de varios conferenciantes que realizan sus discursos en inglés. Además el videojuego se está utilizando como plataforma experimental en el ámbito educativo y, en particular, en una asignatura de inglés de la Universidad de Alicante.

En trabajos más cercanos al nuestro, John Chung Lee (2008) experimentó con el control remoto de la Wii, generando una gran cantidad de recursos y tentativas a seguir, todos ellas enfocados a una conexión y mejor manejo de un Wiimote en el sistema operativo Windows. Como resultado de ello, se obtuvo un lápiz óptico que funciona con dicho dispositivo, capaz de enviar señales al control, el cual interpreta, con un software específico, las señales como si fueran clics encima de una pantalla o proyección. Todas estas investigaciones se han enfocado, exclusivamente en la educación y capacitación e-learning.

En nuestra investigación, comparada con los trabajos citados, se diseñó y se implementó interfaces gráficas de usuario utilizando el folclor ecuatoriano, aprovechando los beneficios de OOHDM y de la IA, aplicando técnicas de razonamiento hacia adelante y hacia atrás en el n-puzzle.

\section{Conclusiones y trabajo futuro}

En esta investigación se diseñó e implemento Puzzlemote, un videojuego didáctico enfocado a niños de 6 a 10 años. Metodológicamente se ini- ció con la investigación descriptiva de videojuegos didácticos, motores de juegos, las fases de la Metodología de Diseño Hipermedia Orientada a Objetos y su aplicabilidad para el desarrollo de videojuegos. Se investigó además las técnicas de razonamiento de Inteligencia Artificial (IA) llamadas encadenamiento hacia atrás y hacia adelante. Luego mediante el lenguaje unificado de modelamiento (UML) se modeló la aplicación 2D, la técnica de IA señalada, y la librería para la manipulación del Control Remoto de la Consola Wii (Wiimote) para usarlo durante el tiempo de juego. Finalmente se implementó la interfaz en dos dimensiones, controlada por este dispositivo y por las reglas de razonamiento provisto por la IA.

Los resultados muestran que este programa ayudó a mejorar el raciocinio lógico y matemático, de niños entre 6 y 10 años, mediante la comprensión y la solución de problemas relacionados a armar rompecabezas. Para validarlo, se planteó a un grupo de estudiantes el reto de armar este tipo de rompecabezas en el menor tiempo posible. En la primera prueba de aplicación durante una hora, no se logró que ningún estudiante resuelva dichos rompecabezas. Luego de un entrenamiento inicial y de proveerles las pautas e indicaciones de cómo resolver estos rompecabezas, se logró despertar el interés en los niños, lográndose reducir los tiempos de resolución a menos de 15 minutos, para los rompecabezas del 8-puzzle y del 15-puzzle, pero no así para el rompecabezas del 24-puzzle el cual a los niños les resultó muy difícil de resolverlo.

Desde el punto de vista de la IA, se pudo comprobar que la combinación de las técnicas de razonamiento hacia adelante y hacia atrás utilizando grafos facilitan que la computadora desarme y arme el rompecabezas con jugadas válidas, memorizando los movimientos iniciales y reconstruyendo esos movimientos invirtiendo la lista enlazada.

Como trabajo futuro se plantea desarrollar este videojuego para dispositivos móviles y plataformas 3D con consolas de videojuegos como Play Station, Nintendo y X-Box, utilizando las mismas 
técnicas de IA planteadas en esta investigación. Además se planea trascender con este tipo de tipo de aplicaciones, también para personas de la tercera edad ya que ayudan al entrenamiento de la memoria y podrían evitar el desarrollo del síndrome de Alzheimer, tal como lo plantean investigadores como los de la Organización norteamericana Puzzles to Remember, donde médicos como Daniel C. Potts, Ellen Woodward Potts, Max Wallack, Carolyn Given, entre otros, han probado que armar rompecabezas ayudan a evitar el deterioro de las neuronas de la memoria de las personas de la tercera edad, y así evitar el desarrollo de dicho síndrome (Wallack, 2013).

\section{Referências}

Álvarez, F. (2013). Uso de videojuegos educativos, caso de estudio: México. Revista Iberoamericana para la Investigación y el Desarrollo Educativo. 10, Recuperado en 2 noviembre, 2013 de http://www.ride.org.mx/docs/ publicaciones/10/juegos_educativos_software/H01. pdf.

Barajas, S. \& Álvarez, R. (2012). Mathematics Game e-Library for Elementary School, Study Case: Mexico. Proceedings of the SPDECE-2012. Ninth multidisciplinary symposium on the design and evaluation of digital content for education, (pp. 123134).

Bello, R. (2002). Aplicaciones de la Inteligencia Artificial. Guadalajara: Universidad de Guadalajara.

Buckland, M. (2004). Programming Game AI by Example. Jones \& Bartlett Publishers: USA.

Doran, J., \& Michie, D. (1966). Experiments with the Graph Traverser Program. Proceedings of the Royal Society, 294, 235-259.

Durkin, J. (1994) Expert Systems: design and development. Macmillan Coll Div.

Espinosa, M., \& Valdivia, Z. (2008). La inteligencia artificial en la informática educativa, Revista de Informática Educativa y Medios Audiovisuales, 5(10), 11-18.

González, J., \& Zapata, G. (2010). Desarrollo de un software educativo para fomentar valores desde la formación personal y social. (Tesis de Licenciatura, Universidad Central de Venezuela, Facultad de Humanidades y Educación, Escuela de Educación).
Illanas, A., Calvo-Ferreret, J., Gallego, F., \& Llorens, F. (2013). Predicting student performance in foreign languages with a serious game. Proceedings of INTED2013 Conference 4th-6th March 2013, (pp. 52-59). Valencia, España. Recuperado en 2 noviembre, 2013 de http://rua.ua.es/dspace/ bitstream/10045/27188/1/1013.pdf.

Illanas, A., (2013). Un videojuego como sistema predictivo de aprendizaje, Memoria para la obtención del Diploma de Estudios Avanzados, (Tesis de Doctorado en Ingeniería Informática y Computación, Universidad de Alicante).

Kurzweil, R. (2010). The Singularity is Near: The movie trailer. Recuperado de http://www.singularity.com/ themovie/\#.UnAiuVMRKSo.

Lee, J. (2008). Hacking the NintendoWii remote. Pervasive Computing, IEEE, 7(3), 39 - 45. doi:10.1109/ MPRV.2008.53.

Lee, J., Hudson, S., \& Tse, E. (2008). Foldable interactive displays. Proceedings of the 21st annual ACM symposium on user interface software and technology, October 2008. (pp. 287-290). doi:10.1145/1449715.1449763.

Levitin, A., \& Levitin, M., (2011). Algorithmic Puzzles. Oxford University Press: USA.

López, F. (2011). Relación entre el hábito de consumo de videojuegos y el rendimiento académico diferencias en género y edad en tercer ciclo de educación primaria, INFAD Revista de Psicología/International Journal of Developmental and Educational Psychology, 1(1), 603612.

Marzal, J., \& Sáez, E. (2013). Videojuegos y Cultura Visual. Ediciones Sociedad Latina de Comunicación Social, La Laguna (Tenerife), 2013 (CAC, Cuadernos Artesanos de Comunicación/42). Recuperado en 2 noviembre, 2013 de http://www.revistalatinacs.org/068/cuadernos/ cac42.pdf.

Millington, I., Funge, J. (2009) Artificial Intelligence for Games (2nd ed.). CRC Press: USA.

Murphy, R., Penuel, W., Means, B., Korbak, C., \& Whaley, A. (2001). E-DESK: A Review of Recent Evidence on the Effectiveness of Discrete Educational Software. Menlo Park, CA: SRI International. Recuperado en 2 noviembre, 2013 de http://ctl.sri.com/publications/ downloads/Task3_FinalReport3.pdf.

Quishpe, H., \& Conde, S. (2010). Incidencia del uso de Software Multimedia en el Aprendizaje de los Niños con necesidades educativas especiales (Tesis de Licenciatura en Informática Aplicada a la Educación, Universidad 
Nacional de Chimborazo, Facultad de Ciencias de la Educación Humanas y Tecnologías, Riobamba, Ecuador).

Rich, E., \& Kevin, K. (1994). Inteligencia Artificial (2nd ed.). McGraw Hill: México, 1994.

Rodríguez, J., Pompa, L., Rodríguez, O., \& Hidalgo, R. (2013). Version 2.0 of the collection of educational games, In 15TH International Convention and Fair 2013, Habana, Cuba.

Rusell, S., \& Meter, N. (1996). Inteligencia Artificial: un enfoque moderno. Prentice Hall: México.

Selver, S. (2004). Using nintendo wii remote control from finger tracking, gesture detection and as HCI device. Instituto de Sistemas de Información y Medios Computacionales de la Universidad Tecnológica de Graz, Austria. Recuperado en 2 de noviembre 2013 de http://202.28.94.62/webprojectcs/cs2553/gr57/ Download_files/seminar_is.pdf.

Shneiderman, B., \& Plaisant, C. (2006). Diseño de interfaces de usuario: estrategias para una interacción persona-computadora efectiva. México: Addison Wesley.

Silva, D., \& Mercerat, B. (2001). Construyendo aplicaciones web con una metodología de diseño orientado a objetos. Revista Colombiana de Computación - RCC, 2(2). Recuperado en 2 de noviembre 2013 de http:// revistas.unab.edu.co/index.php?journal $=$ rcc\&page $=$ article\&op=view\&path\%5B\%5D=1116

Wallack, M., \& Given, C. (2013). Why did grandma put her underwear in the refrigerator? an explanation of alzheimer's disease for children. CreateSpace Independent Publishing Platform: USA.

\section{Puzzlemote: Wii remote controller videogame for 6 to 10 years old children}

\section{Abstract}

Introduction: Educational video games help to create meaningful learning situations for discovery, which may bring problem-solving skills and decision making. This research presents the design, implementation and evaluation of a game-based educational software called "Puzzlemote" aimed at children from 6-10 years. Method: It was used the Hypermedia Design Methodology Object Oriented (OOHDM) to create a fun and interactive environment, and Artificial Intelligence (Al) that promotes logical thinking and complex problem solving. The algorithm used is based on reasoning techniques that implemented forward and backward features to scramble the puzzle so set it in order, which differs this approach from similar others, where it is common to use the $A^{*}$ algorithm . For its implementation it was employed the Wii Remote (Wiimote) controls, bearing in mind that the user could have a richer experience with the software connected to this device during the game time. Results: The results show that this program improves the understanding and the logical reasoning demanded to problem solving, especially the puzzle known as n-puzzle, for children who were in the primary stage of education. Conclusions: It was found that the combination of reasoning techniques using the computer graph helps to disassemble and assemble the puzzle when valid moves were made, memorizing the initial movements and rebuilding these movements by reversing a linked list. As future work is proposed to develop this game for mobile devices and $3 D$ platform gaming consoles such as Play Station, Nintendo and $X$-Box. It is also planned to go beyond with this kind of application, providing an approach for elderly people due to the memory training which could prevent the development of Alzheimer's Syndrome.

\section{Keywords}

Learning. Video games. Educational games. Object Oriented Hypermedia Design Method. Artificial intelligence. Wii.

Recibido: Octubre 9, 2013

Aceptado: Noviembre 3, 2013 
Acerca de los autores:

\section{Marcelo Daniel Torres Vinueza}

Bachiller en Ciencias, Ciencias Básicas - Unidad Educativa La Salle/Quito, Bachiller en Ingeniería - ESPE. Gerente de TI - Junta Nacional de Defensa del Artesano.

marcextv@outlook.com

\section{Walter Fuertes}

Ingeniero de Sistemas y Informática - ESPE, Máster en Informática - EPN. Profesor-Investigador - ESPE.

wmfuertes@espe.edu.ec

\section{César Xavier Villacís Silva}

Ingeniero de Informática y de Sistemas - ESPE, Máster en Sistemas Multimedia y Informática - ITI/Quito, Diplomado em Diseño Curricular por Competencias - ESPE. Docente ESPE/DCC.

cjvillacis@espe.edu.ec

\section{Margarita Elizabeth Zambrano Rivera}

Ingeniera en Sistemas e Informática - ESPE. Docente - ESPE/ DCC.

mzambrano@espe.edu.ec

\section{Carlos Teiron Prócel Silva}

Ingeniero Electrónico - ESPE, Máster el Seguridad y Desarrollo - IAEN/Quito, Máster en Ciencias de la Computación - IME/ Rio de Janeiro, Diplomado en Gestión Directiva - ESPE. Docente - ESPE/DCC.

cprocel@espe.edu.ec

Para citar el artículo:

TORRES VINUEZA, M. D. ; FUERTES, W.; VILLACÍS SILVA, C. X. ; ZAMBRANO RIVERA, M. E. ; PRÓCEL SILVA, C. T. Puzzlemote: videojuego controlado con el mando de la Wii para niños de 6 a 10 años. AtoZ: novas práticas em informação e conhecimento, Curitiba, v. 2, n. 2, p. 94-105, jul./dez. 2013. Disponível em: <http://www.atoz.ufpr.br>. Acesso em: 